came, had no memory whatever for the events of the last ten days, took her food well, and sat up in bed.May 12th: Talks a good deal about religious matters, but has no delusions; went out to-day on a month's trial. June 12th: Was discharged this day, recovered.

The following is the history as given by the patient. She has always been hysterical. No history of intemperance or insanity in the family. For several years she has been a diligent student of the Bible, and thoroughly believed everything therein regarding a future state. One month before admission two of her children were taken ill with influenza, after their recovery she herself contracted the disease, and had a most severe attack. The pain in her head was excruciating, and she was quite prostrate in mind and body for two weeks. After spending an anxious day she relapsed into a deep sleep, during which she had a dream, and was awakened by a loud voice, which said, "You are dead." She felt quite helpless, and lay in this state for two days, absolutely refusing all food. When visited by the doctor she informed him she was dead, and wished to be buried. She was at once removed to this asylum as insane. She remembers coming into the gates of the grounds, which she thought was the cemetery. She says that had she been put into a grave she could have offered no resistance.

Remarks.--Some very interesting clinical points arise in this most unusual case. The condition of trance is exceedingly rare in this country, Gowers having seen only four cases. Regarding the diagnosis of the case, I am not yet satisfied as to the true mental condition. Here is a woman whose physical and mental powers are exhausted with the care and anxiety of nursing her sick children, then she is herself prostrated by a severe physical illness with great mental depression. She is not predisposed to insanity either by hereditary transmission or otherwise, but she is undoubtedly hysterical and emotional. Was the woman insane? The voice which she heard was not a true hallucination, as she was unconscious from sleep at the time. Legally, she was insane without a doubt; she was not responsible for her actions, and would have probably died from want of food, the diagnosis thus resting between (1) delusional insanity, (2) hysteria, (3) catalepsy, and (4) trance. She was not suffering from catalepsy, as evidenced by the absence of muscular rigidity; nor were the symptoms purely hysterical, as shown by the utter impossibility to restore her to consciousness. Then regarding insanity pure and simple, this is negatived by the fact that she has no memory whatever for what occurred during those ten days, and the sudden and complete recovery from all the symptoms. I am inclined to think that this was a case in which, from severe nervous exhaustion and with a predisposition to emotion, the patient's mind was temporarily unhinged, and that the trance condition was due to an inhibition or arrest of action of the nervecells, probably from previous exhaustion. Portsmouth.

\section{REMARKS ON MODERN METHODS OF CATARACT EXTRACTION. ${ }^{1}$}

BY CHARLES G. LEE,

HON. SURGEON TO THE LIVERPOOL EYE AND EAR INFIRMARY.

THE method usually adopted for the removal of an opaque senile lens is pretty well known, and were it not that just now the minds of ophthalmic surgeons are much exercised as to the advisability or otherwise of one or two proceedings in the course of the operation, I should not have presumed to call attention to a subject that at first sight might appear commonplace. In furnishing a title for this short paper, I have made use of the word "modern," to indicate my desire to discuss the question from a clinical and experimental, rather than from a historical, standpoint, believing that by this method any personal experience one may have obtained will be more easily and expeditiously brought under notice. I think we are justified in applying the term "modern" to the operation known as von Graefe's, and to all the modifications that have been subsequently introduced, very few operators adhering strictly to the letter of that great master's directions.

Read before the Chester Medical Society, April 11th, 1890.
Previously to Graefe's time the flap operation held almost universal sway, having supplanted the older operation of couching. I think it well to divide the steps of the operation into five, as each modification or novelty can then very readily be discussed in its appropriate place.

First stage: incision.-The modern tendency is to place this more forward than in the original directions, the causes being chiefly two- $(a)$ danger of escape of vitreous and $(b)$ fear of wounding the ciliary body. Personally, plan my line of incision similarly to that adopted by Mr. Swanzy, and I cannot better describe it than by quoting from his recent handbook: "The point of the knife is entered just in the margin of the clear cornea, at the outer extremity of a horizontal line which would pass three milli. metres below the summit of the cornea. This line is easily found by placing the knife, which is about two millimetres broad, horizontally across the cornea, so that a margin of clear corneal tissue one millimetre broad may remain exposed between the knife and the summit of the cornea. The knife is then passed cautiously through the anterior chamber and the connter-puncture made in the corneal margin at the inner extremity of the horizontal line described, and the incision finished in the corneal margin by a few slow to-and-fro motions of the knife." There is considerable variation as to the situation of the corneal incision, the general tendency, however, being to advance it. Mr. Pridgin Teale, using a knife somewhat resembling a Beer's, places his line of incision midway between the centre and periphery of the cornea.

Second stage: iridectomy.-We now enter upon most debatable ground. This procedure, which was formerly held to be a most essential part of the operation, has of late years fallen into disuse in the hands of some most eminent operators. The chief reasons for this abandonment of an iridectomy seem to be, first, that it is unneces. sary; secondly, that from a cosmetic standard the results are not so good. We may at once concede the possibility of removing the hardened nucleus without an iridectomy, but it may be that only then have one's difficulties com. menced; frequently we are met by an immediate prolapse of the iris into the corneal wound, which resists all attempts at reduction, and it must be excised. Should this accident not happen at this stage, it is by no means certain that it will not occur-indeed, my experience points to the third or fourth day after the operation as those on which it may happen. The mode in which it occurs is worth notice: it appears to be coincident with the closure of the corneal wound; the filuration of the aqueous being stopped, the iris is bulged by the retained fluid, and it only requires a slight movement on the part of the patient to break through the recently cemented lymph-a sudden gush of aqueous occurs, and the iris is carried into the wound. To free the iris from its incarceration is now no easy task, and if it remain in its malposition our patient has probably to encounter the dangers of sympathetic ophthalmitis. To these very obvious and immediate complications must be added those that are liable to arise from the bruising and stretching the iris has to undergo during the passage of the lens through the pupil, and whose advent may be looked for from the seventh to the tenth day. Although we have not yet exhausted the adverse possibilities that must be weighed before we decide to adopt the socalled simple method of extraction, their enumeration is scarcely necessary when we consider the very striking admission made by $\mathrm{Mr}$. Teale at the Leeds meeting last year, when he said: "Given a patient with only one eye, and that one affected with cataract, I should perform an iridectomy, being content to forego a little extra brilliancy in result to greater probability of successful operation." Knowing that Mr. Teale was one of the very earliest of British surgeons to relinquish iridectomy, and has had perhaps more experience than any other oculist of this opera. tion, it appears clearly our duty to give our patients the best chance for recovery of a sound eye, and this by iridectomy.

The third stage-laceration of capsule-I only stay to mention this stage.

Fourth stage: delivery of lens. - This is accomplished by a gentle pressure applied to the lower edge of the cornea in some few cases, owing to the corneal incision being rather small, or from some other cause, the lens sticks at its equator, and to avoid undue pressure I have used the tractor which is figured below. It was made for me by 
Messrs. Weiss, and its application is as simple as it has been gratifying.

Fifth stage, usually termed " the toilette of the wound," includes much more than might be presumed from its designation. Not simply is it concerned in clearing the edges of the incision from blood and débris, but also freeing the area of the pupil from any soft lenticular matter that is apt to remain, and give rise to secondary cataract. Various expedients are employed to remove these particles of cortical matter. Great reliance is placed upon digital or instrumental pressure applied either directly to the cornea, or from the outside of the lid. A grave objection to this procedure exists in the fact that even when it is most delicately conducted a rupture of the hyaloid not infrequently occurs, and escape of vitreous follows ; besides, it is often ineffectual. To Dr. McKeown of Belfast belongs the credit of introducing a novel and most effective method of clearing away cortex - viz., by introducing fluid into the capsule of the lens. He published his first series of cases in 1884. The instruments he recommended for injection of the fluid were hollow needles and syringes. Early in 1886 I used McKeown's syringe on two occasions, and these sufficed to show me that, whilst we had a most useful line of practice indicated, the means used for carrying it out were unsatisfactory. Accordingly I replaced the jerky and unsteady flow of the syringe by the equable and controllable flow obtained by a very simple irrigation apparatus. This was fully described at the Brighton meeting of the British Medical Association in 1886. Since then I have continued to use the same simple apparatus, having, however, slightly modified the terminal. The accompanying figure shows the one now in use. I have employed irrigation on more than thirty occasions, always with satisfaction and, I believe, with benefit. The thorough-
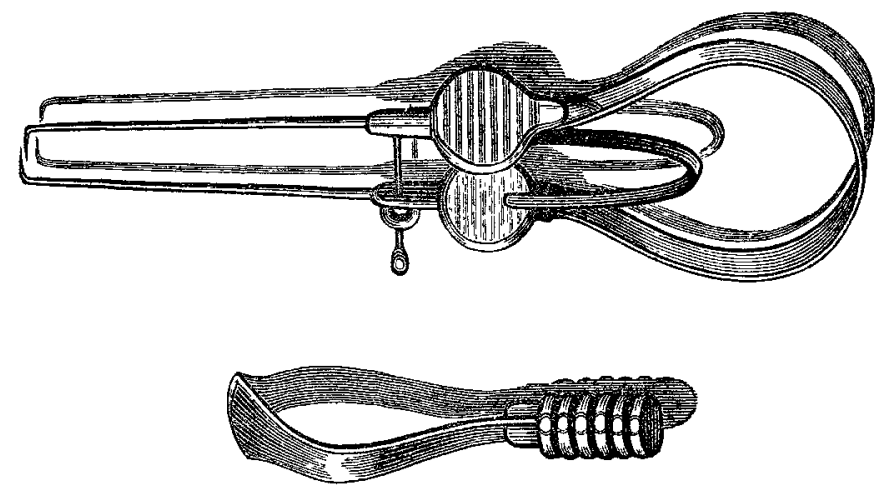

ness with which the irrigator clears away the soft matter is really remarkable. Having by this method secured a black coloboma, and the patient being able to count fingers held in front of the operated eye, it only remained to clear the conjuctival sac from any débris of blood \&c. The outside of the eyelids are usually dredged with iodoform powder, and double pads of Gamgee's lint secured over each eye with strips of plaster. Finally, a roller bandage is fixed over all, the patient removed to bed, where he remains till the eighth day. Many ophthalmic surgeons do not disturb the dressings for a week, unless warned by pain that something is amiss; but as I have seen more than one cornea slough painlessly, it is my custom to remove the pads the day after the operation and replace them by clean ones; the eyelids need not be opened unless there is some redness, swelling, or discharge visible. Should there be anything to arouse suspicion, the lids are opened and an inspection of the wound is made; if now, or at a later period, there are any signs of sloughing, we irrigate the whole of the anterior chamber with an antiseptic fluid, and believe that by these means we have saved from utter destruction one case at least which had the ostrichlike policy of trusting all was going on right because we could not see the untoward processes that had already commenced would undoubtedly have been destroyed.

The judicious application of antiseptics to ophthalmic surgery has long been aimed at by oculists; at the Liver pool Eye and Ear Infirmary we use a solution of biniodide of mercury known as "panas." The instruments to be employed in the operation are immersed in this solution, and the patient's face, as well as the conjunctival cul-de-sac, are thoroughly laved with it.

In conclusion, I beg to acknowledge the kindness and skill of Mr. H. E. Jones, assistant surgeon to the Liverpool Eye and Ear Infirmary, in making sketches of the instruments. Iiverpool.

\section{ON A CASE OF POISONING BY NICOTINE.}

BY G. STILLINGFLEET JOHNSON, M.R.C.S., F.C.S., DEMONSTRATOR OF CHEMISTRY AT KING'S COLLEGE, LONDON.

A TEMPERATE man, aged seventy-five, was found on a doorstep with his throat bleeding, having thrown from him a bottle, and with a greenish vomit on the ground near him. A paper was in his hand, bearing the words "I did this myself." A knife was also near him. He was taken to King's College Hospital, where the bleeding was found to proceed from a divided external jugular vein, which was at once ligatured. Preparations were then made to empty the stomach, but during attempts to adjust the tube of the stomach-pump, which the man resisted actively, he suddenly died, without convulsions. At the necropsy there were no signs of the action of any irritant poison, and the only abnormality observed in any organ was a slight degree of mitral constriction. This being so, and the amount of blood lost being, in the opinion of the house surgeon, quite insufficient to account for death, the stomach and its contents were preserved, and by order of the coroner, Dr. J. Troutbeck, were brought to me for chemical examination. I received the stomach, tied at the cesophageal and pyloric orifices, also a small piece of small intestine, tied at each end; the whole immersed in spirits of wine in a well-stoppered glass bottle. The alcoholic liquor was reserved for separate examination. The contents of the stomach measured rather more than 2 fluid oz. (60 cubic centimetres). It was a brown grumous fiuid, with a feebly acid reaction to litmus paper. It was easily filtered through paper, the insoluble matter being preserved for further examination. The clear filtrate was tested for poisonous metals with a negative result, but it gave the brown precipitate with a solution of iodine in iodide of potassium, characteristic of a vegetable alkaloid. The clear liquid was now rendered slightly alkaline with ammonia, stirred and set aside for a time to favour precipitation. A flocculent precipitate formed at once after adding the ammonia, but did not appear to increase on standing. The precipitate was now separated by filtration, and well washed with cold water. The clear ammoniacal filtrate was tested for alkaloids by acidulating a small portion with hydrochloric acid and adding a solution of iodine in potassium iodide. The alkaloidal precipitate formed as abundantly as ever. On the other hand, the precipitate produced by addition of ammonia to the fluid from the stomach after solution in hydrochloric acid gave only a slight opalescence with solution of iodine in potassium iodide. These results indicate the existence of a soluble vegetable alkaloid. In order to separate the soluble alkaloid from the other sub. stances in the watery solution the clear ammoniacal filtrate was shaken with chloroform. The chloroform separated fairly well from the watery solution, from which it was drawn off by means of a stoppered funnel, and evaporated cautiously over steam. A yellowish oil remained in the dish, whose odour, especially when the dish was warm, was highly penetrating, stinging the nasal membrane like ammonia, and recalling the odour of a foul tobacco pipe. The weight of this oil obtained from 30 c.c. (= one fluid ounce), or half of the stomach contents, was 3 centigrammes $\left(=\frac{46}{100}\right.$ ths of a grain). The oil dissolved almost entirely in water, only a little resinous matter remaining, and its aqueous solution reaction was intensely alkaline to litmus. The aqueous solution was acidulated with hydrochloric acid, and the acid liquid evaporated to dryness over steam. The residue gave an acid solution to water, so that the base forms a hydrochloride. A portion of the solution of the hydrochloride of the alkaloid was rendered alkaline with potash, and the solution was warmed; a strong tobacco-like odour was developed. A second portion of the solution of the hydrochloride of the alkaloid was mixed with an aqueous solu. tion of mercuric chloride, when immediately a copious white precipitate formed, which dissolved on being warmed, and deposited in well-formed crystals on cooling. This reaction proves the absence of coniine, which gives an amorphous precipitate with $\mathrm{HgCl}_{2}$. The above reactions all indicate that the soluble oily alkaloid is nicotine.

I now turned my attention to the alcoholic liquor in which the stomach had been preserved. The filtered liquid was evaporated to dryness over steam, when a copious solid residue remained. The residue was extracted with acidulated water, and the solution gavestrong indication of the presence G 2 